\title{
"The Effervescent Gallbladder": A Rare Ultrasonographic Finding that Reflects the Presence of Gas within the Gallbladder
}

Authors

Affiliations
Á. Lozano Rodríguez ${ }^{1}$, R. Fuentes Pavón ${ }^{1}$, P. Junguera Rionda ${ }^{1}$, P. Alemán Flores ${ }^{1}$, A. Fuentes González ${ }^{2}$, C. López Gutiérrez , D. Batista Martín

${ }^{1}$ Radiología, Complejo Hospitalario Universitario Insular Materno-Infantil, Las Palmas de Gran Canaria, Spain ${ }^{2}$ Complejo Hospitalario Universitario Insular Materno-Infantil, Service of Anaesthesiology and Resuscitation, Las Palmas de Gran Canaria, Spain
Key words

- gallbladder

ultrasound 2d

infection

\section{Abstract}

This article deals with the "effervescent gallbladder", a rare ultrasonographic finding indicative of

\section{Introduction}

Emphysematous cholecystitis is a rare and severe variant of acute cholecystitis, characterized by gas production in and around the gallbladder wall [1]. This entity has an insidious onset, which often leads to delayed diagnosis with consequently high associated morbidity and mortality rates. In this article, we review the ultrasonographic signs associated with emphysematous cholecystitis with special attention to the socalled "effervescent gallbladder". This sign consists in the ultrasonographic finding of tiny echogenic foci rising like bubbles in a glass of champagne and floating from the dependent to the nondependent portion of the gallbladder [2]. We postulate that, although indicative of the presence of air within the gallbladder, this sign remains vastly underreported. Here we describe 3 cases of emphysematous cholecystitis, where this unusually reported finding was identified and documented with photographs.

\section{Correspondence}

Álvaro Lozano Rodríguez, MD Radiología

Complejo Hospitalario Universitario Insular Materno-Infantil

Av. Marítima del Sur

Las Palmas de Gran Canaria Spain 35000

Tel.: + 34/928/441335

alvaro_Ir@icloud.com

\section{nenation}

\section{Case Reports \\ $\nabla$}

\section{Case 1}

A 53-year-old male patient with a history of type II diabetes and diabetic nephropathy presented with abdominal pain in the right lower quadrant, which radiated to the right upper quadrant. The Blumberg's sign was positive and the results of blood tests indicated leukocytosis (20600). An ultrasonographic study revealed a hyperechoic linear image within the gallbladder with dirty shadowing and shift phenomena, suggestive of the presence of gas within the gallbladder. 3 cases are described and illustrated with photographs. Possible causes are reviewed and discussed.

the presence of gas ( $\bullet$ Fig. 1). Additionally, multiple punctate echogenic foci were observed moving from the dependent to the non-dependent portion of the gallbladder and merging into the mentioned linear image. CT scan confirmed the presence of gas within the gallbladder. Both the pericholecystic fat adjacent to the hepatic flexure and the perihepatic fluid showed signs of inflammation. The patient was diagnosed with peritonitis secondary to emphysematous cholecystitis and was subjected to surgery, whereby the ultrasonographic findings were confirmed. A subsequent pathological study evidenced gangrenous cholecystitis.

\section{Case 2}

A 75-year-old female patient with a history of diabetes mellitus and hypertension was referred to our service with a diagnosis of acute cholecystitis. She presented with nausea, vomiting and shivering. Laboratory tests showed a significant rise in the amount of cholestatic enzymes GGT and alkaline phosphatase (1113 and $1404 \mathrm{mU} /$ $\mathrm{ml}$, respectively). Hemogram results were within normal limits. The ultrasonographic findings were similar to those of case 1 ( $\bullet$ Fig. 2 ) although gallbladder lithiasis and pneumobilia were additionally observed. The patient underwent surgery and a subsequent pathological study showed signs of acute and chronic cholecystitis.

\section{Case 3}

A 79-year-old male patient with a history of fever that remitted with antibiotic treatment was referred to our service for evaluation of a solid liver lesion. The results of all laboratory tests 

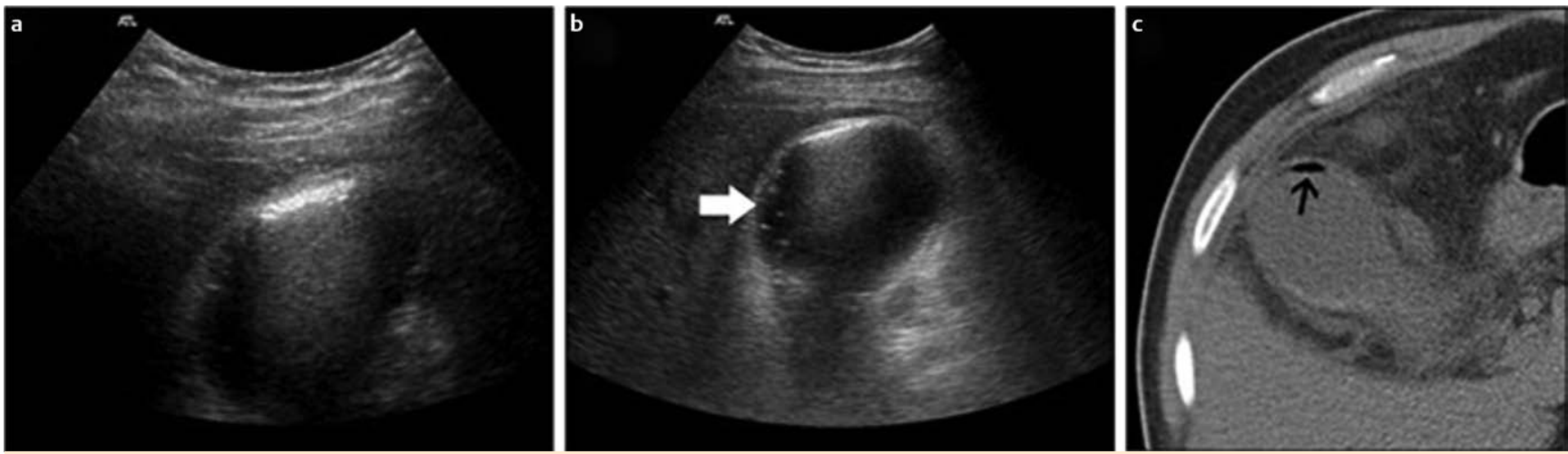

Fig. 1 a Hyperechoic linear image with dirty shadowing and shift phenomena suggestive of the presence of gas within the gallbladder. b Multiple punctate echogenic foci moving from the dependent to the nondependent portion of the gallbladder (white arrow) and merging into the mentioned linear image. c CT scan confirmed gas (arrow).
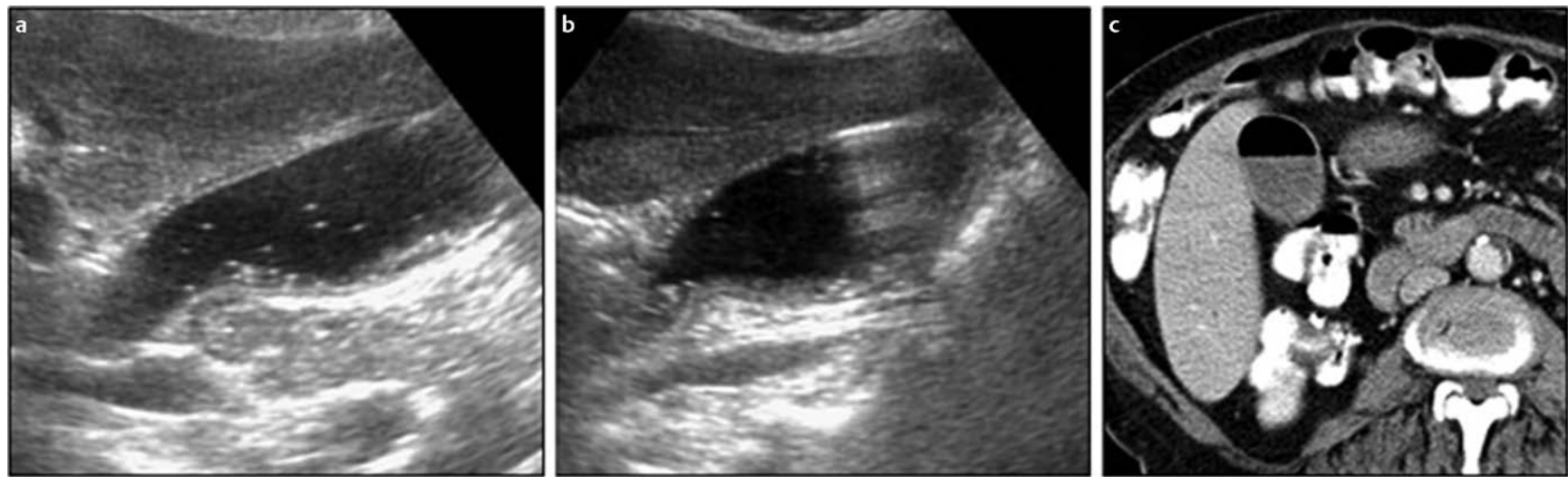

Fig. 2 a Multiple punctate echogenic foci slowly emerging from the dependant portion of the gallbladder. $\mathbf{b}$ Linear hyperechogenic image with dirty shadowing at the top of the lumen. c CT scan showed an airfluid level in the gallbladder that confirmed the presence of intraluminal gas.
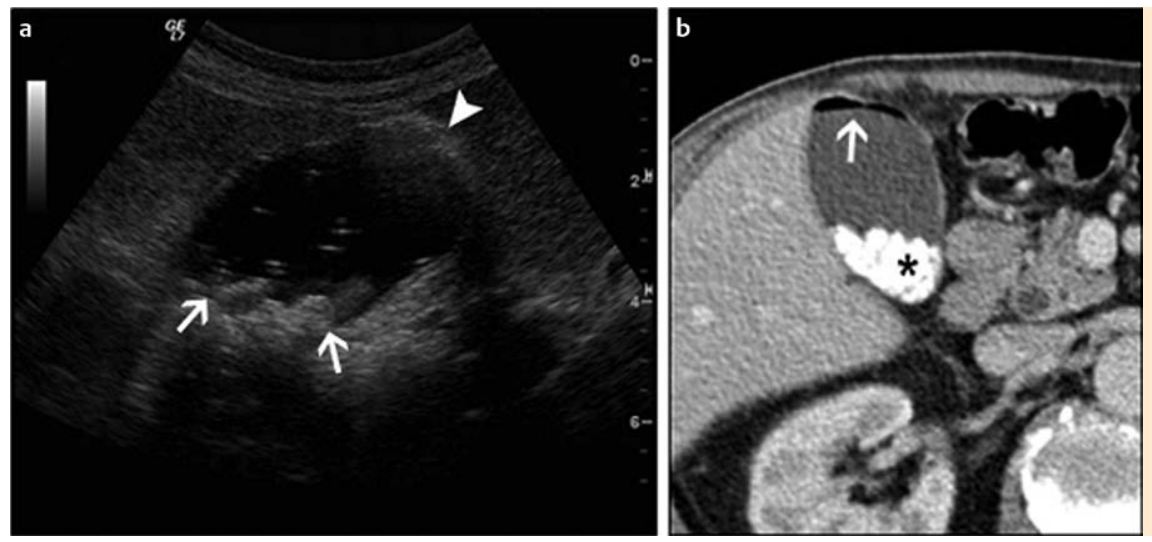

Fig. 3 a Ultrasound image showing cholelithiasis (arrows). Gas within the gallbladder was suspected from mobile refracting foci and echogenic linear image (arrow tip). b CT scan confirmed the presence of gas (arrow) and lithiasis (asterisk).

were within normal limits. Ultrasonographic examination revealed cholelithiasis and the presence of air within the intrahepatic bile ducts, the cystic duct and the gallbladder lumen. Mobile refractive foci similar to those described above (cases 1 and 2 ) were detected in the gallbladder ( $\bullet$ Fig. 3 ). Additionally, a heterogeneous $3 \times 4 \mathrm{~cm}$ predominantly cystic lesion with membranes and calcifications was identified in hepatic segment II-III, which was suggestive of hepatic hydatid disease. The treatment consisted of resection of segment II-III and cholecystectomy. A subsequent pathological study confirmed the presence of a partially calcified hydatid cyst which communicated with the bile ducts. Cholestasis, sclerosing cholangitis and chronic lymphoplasmacytic inflammation with abundant eosinophils were also found within the liver parenchyma adjacent to the hydatic cyst. The gallbladder showed signs of chronic cholecystitis and cholelithiasis without signs of acute cholecystitis.

\section{Discussion \\ $\nabla$}

Cholelithiasis is the main cause of acute cholecystitis. The prevalence of acute cholecystitis is approximately $5 \%$ of patients presenting with acute abdominal pain at emergency centers. Traditionally, this entity has been diagnosed on the basis of the clinical triad: right upper quadrant tenderness, elevated body 
temperature and elevated white blood cell count. However, this triad is currently known to be present in only $8 \%$ of patients [3]. Emphysematous cholecystitis is a variant of acute cholecystitis, where gas-forming bacteria are involved. Clostridium welchii and Escherichia coli have often been identified in patients with this condition [4-6]. The entity was first described by Welch and Flexner in 1896 [7]. Stolz also reported it in 1901, after performing an autopsy [1]. Its associated radiologic signs were first described by Hegner in 1931 [8]. Its clinical definition is based on the presence of air in the gallbladder lumen, wall or tissues surrounding the wall, in the absence of abnormal communication with the gastrointestinal tract $[4,6]$. This condition affects men twice as frequently as women. Conversely, cholecystitis is twice more frequent among women than among men [5]. Most patients are between 50 and 70 years old and $30-50 \%$ of them have a history of diabetes mellitus and peripheral atherosclerotic disease (like in cases 1 and 2 in this article). Vascular compromise of the cystic artery is thought to play a significant role in the development of emphysematous cholecystitis.

Emphysematous cholecystitis is associated with a higher prevalence of acalculous disease and gallbladder perforation than acute cholecystitis $[4,6]$ (in case 1 above, cholelithiasis was not found). It is also associated with a high risk of suffering other complications, including gangrene, perforation, pericholecystic abscess and bile peritonitis [4] (like in case 1), all of which lead to higher mortality rates (15\%) than acute cholecystitis (4\%) [2] and make early diagnosis and surgery essential to improve patient survival. The onset is often insidious and the condition may progress quickly, thus requiring emergency surgery $[2,5,6,9]$. Since leukocytosis is not found in about $50 \%$ of patients (like in cases 2 and 3 above), the diagnosis of emphysematous cholecystitis often requires imaging techniques. The radiological diagnosis is based on the detection of gas within the gallbladder (stage 1), the gallbladder wall (stage 2 ) or the pericholecystic tissues (stage 3). CT scan is considered to be the gold standard for the detection of emphysematous cholecystitis. However, due to the insidious onset of the clinical picture, the use of this technique is often delayed during the first few hours. When gallbladder gas is suspected from abdominal X-ray in the absence of abnormal communication between the biliary system and the gastrointestinal tract, differential diagnosis should be considered against overlying bowel gas, hepatic abscess, retro-peritoneal air and focal biliary lipomatosis [1,5]. Other common causes of gas within the gallbladder include: enterobiliary surgical anastomosis, incompetent Oddi sphincter, spontaneous internal biliary fistula (gallstone perforation, tumor or traumatic fistula), drug effects (magnesium sulfate, atropine, nitroglycerin) and cholangitis [6]. For example, in our case 3 the presence of air within the gallbladder and bile duct could have been accounted for by cholangitis or by a partial response to antibiotic therapy. Only occasionally, air within the gallbladder is detected on X-ray images. For 2 of our patients (cases 1 and 3), $\mathrm{X}$-ray images were normal. In a further post-diagnosis revision of these images specifically searching for signs of gallbladder gas, no evidence was found.

In general, ultrasonographic images show stages 1 and 2 of emphysematous cholecystitis as high reflectivity echoes with distal reverberations and a circular pattern of poorly marginated shadowing typical of gas in the gallbladder fossa outlining the gallbladder wall [6], "dirty shadowing" [5]. Intraluminal gas occupies the upper portion of the gallbladder and can be observed to move as the patient changes position $[4,13]$. Both of these findings were observed in our 3 reported cases. Intramural gas may also appear as an arch-shaped hyperechogenic area on the surface of the gallbladder. This finding might be misinterpreted as a porcelain gallbladder, a different entity which is however easily diagnosed through abdominal X-ray [4-6]. Similar ultrasound images can be observed in the presence of duodenal gas. However, in most of such cases the gallbladder can be identified through the intercostal spaces, which prevents misdiagnosis [4,10-12].

In emphysematous cholecystitis, real-time ultrasound shows punctate echogenic foci without acoustic shadowing in the gallbladder, as they move from the dependent wall to the top, mimicking the bubbles in a glass of champagne $[2,13,14]$ (० Fig. 1-3). Although rarely reported, this finding is pathognomonic of gallbladder gas (and it was present in our 3 patients). In our opinion, rather than being rare, this sign is possibly being underreported. Actually, a specific literature search yielded only 5 articles where this finding had been described [2,5,13-15]. We postulate that misinterpretation of emphysematous cholecystitis-associated ultrasound images could lead to underreporting. Possible sources of confusion are: mistaking bubbles for floating cholesterol crystals in the gallbladder [16] (although crystals tend to be smaller and in our experience move very slowly and differently from champagne bubbles), interpreting images as corresponding to those of a bilio-enteric fistula (although in such cases, bubbles should be expected to emerge from a single point on the communication site instead of emerging from a wide area on the dependent wall $[2,14])$, or interpreting that bubbles correspond to gas release from gas-containing gallstones (although in that case, bubbles should be observed to emerge from the gallstones, not from the wall [15]).

In summary, since the "effervescent gallbladder" appears to be an unequivocal sign of gas in the gallbladder, it could contribute to an early diagnosis of emphysematous cholecystitis. Upon observation of this sign, we recommend careful examination of the gallbladder, specifically looking for the site where bubbles emerge.

\section{References}

1 Stolz A. Uber gasbildung in der Gollinweger. Arch Pathol Anat 1901; 165: 90-123

$2 \mathrm{Wu}$ CS, Yao WJ, Hsiao CH. Effervescent gallbladder: sonographic findings in emphysematous cholecystitis. J Clin Ultrasound 1998; 26: 272-275

3 Stoker J, van Randen A, Laméris $W$ et al. Imaging patients with acute abdominal pain. Radiology 2009; 253: 31-461

4 Bloom RA, Libson E, Lebensart PD et al. The ultrasound spectrum of emphisematous cholecystitis. J Clin Ultrasound 1989; 17: 251-256

5 Grayson DE, Abbottt RM, Levy AD et al. Emphysematous infections of the abdomen and pelvis: A pictorial review. Radiographics 2002; 22: $543-561$

6 Gill KS, Chapman AH, Weston MJ. The changing face of emphysematous cholecystitis. The British Journal of Radiology 1977; 70: 986-991

7 Welch WH, Flexner S. Observations concerning the Bacillus aerogenes capsulatus. J Exp Med 1896; 1: 5-45

8 Hegner CF. Gaseous pericholecystitis with cholecystitis and cholelytiasis. Arch Surg 1931; 22: 993-1000

9 Bernstein D, Soeffing J, Daoud YJ et al. The obscured gallbladder. Am J Med 2007; 120: 675-677 doi:10.1016/j

10 Lorenz RW, Steffen HM. Emphysematous colecystitis: Problems and differential diagnosis of gallbladder gas accumulations. Hepato-gastroenterol 1990; 37 Suppl II: 103-106

11 Konno K, Ishida $\mathrm{H}$, Naganuma $\mathrm{H}$ et al. Sonographic findings. Abdom Imaging 2002; 27: 191-195 doi:10.1007/s00261-001-0054-3

12 Hunter ND, Macintosh PK. Acute emphyshemaous cholecystitis: an ultrasonic diagnosis. AJR 1980; 134: 592-593 
13 Nemcek AA, Gore RM, Vogelzang RL et al. The effervescent gallbladder: A sonographic sign of emphysematous cholecystitis. AJR 1988; 150: $575-577$

14 Lim JH. The effervescent gallbladder. AJR 1988; 151: 612
15 Niederhauser BD, Atwell TD, Maccarty RL et al. Gas-containing gallstones as a cause of the "effervescent gallbladder" sign and pneumobilia. J Clin Ultrasound 2012, Dec 1 doi:10.1002/jcu.22020

16 Yeh HC, Goodman J, Rabinowitz JG. Floating gallstones in bile without added contrast material. AJR 1986; 146: 49-50 